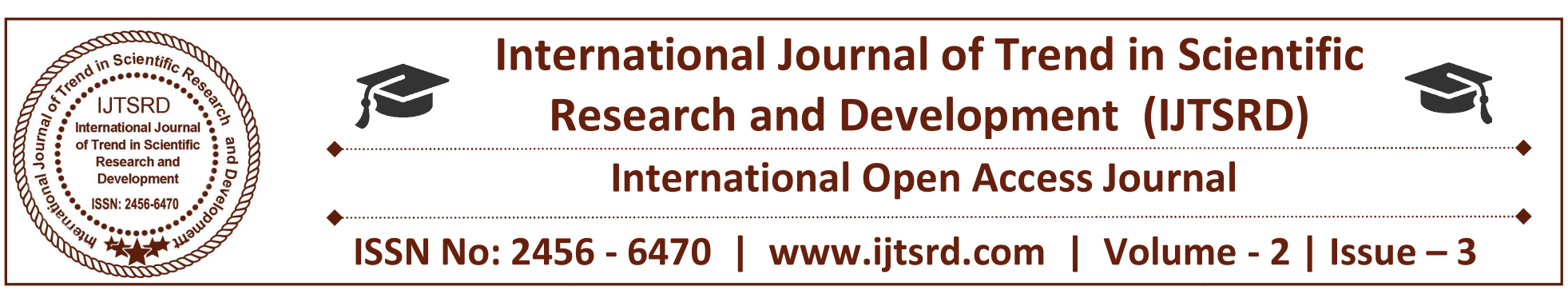

\title{
Vehicle Density Based Traffic Control System
}

\author{
Anand Gaikwad, Shreya, Shivani Patil \\ BE Student, Department of Electronics and Telecommunication, \\ Sinhgad Academy of Engineering, Kondhwa, Pune, Maharashtra, India
}

\section{ABSTRACT}

In this paper, we proposed a system i.e. vehicle density based traffic management system. Present day traffic signal system is fixed time based which may render inefficient if one lane is operational than the others. To optimize this problem we have made a framework for an intelligent traffic control system. Sometimes higher traffic density at one side of the junction demands longer green time as compared to standard allotted time. Basically, it is divided into two modules. In first module, the timing of signal will change automatically on sensing the traffic density at any junction. We, therefore propose here a mechanism in which the time period of green light and red light is assigned on the basis of the density of the traffic present at that time. And in second module, to reduce the crime we develop a technique of automatic number plate recognition using image processing. In this paper, the first section is introduction, second section is literature survey, third section is block diagram and components, fourth section is prototype implementation, fifth section is conclusion, sixth section is references. whatever the result is shown in this paper is partially executed.

Keywords: Vehicle Density, Traffic, Signal

\section{Introduction}

Now-a-days conventional traffic light system is based on fixed time concept allotted to each side of the junction which cannot be varied as per varying traffic density [1]. This causes wastage of time and traffic congestion. The major cause of traffic jamming is the more number of vehicle which is caused by the over growing population. And another problem is that, traffic police has to keep concentration on vehicles every day and at every time. The accuracy to catch the appropriate criminal on the spot is very rarer by traffic police. The signal timing changes automatically on sensing the traffic density at the junction.

Most of the work detects edge of the vehicles and counts the number of traffic on the road. With the help of dedicated algorithm, morphology and image needed to avoid traffic congestion Also we have used ANPR which is an image processing technology used to store the images captured by the cameras as well as the text from the number plate [2].Thus, we have designed a framework for a dynamic and automatic traffic light control system and developed a simulation based model with codes in to help build the system. In this, we propose three approaches. In the first approach - to take data or input from camera. In the second approach - to process the input data using ARM processor and finally display it on the traffic light signal to control the Closed Loop System. We are proposing such a system that compact with such kind of problem by automatically switching the signal by calculating the time at which the vehicles inwards at stop line.

The traffic lights that are in widespread use today do not do much complex reasoning when deciding when to change the lights for the various road users waiting in different lanes. How long the signal stays green in one lane and red in another is most often determined by simple timing that is calculated when the crossing is designed. Even though today's methods are robust and work well when the traffic load is distributed evenly across the lanes in the intersection, the systems are very inefficient because they are unable to handle various simple situations that arise throughout the day. Unnecessary waiting time in the signal can be avoided by determining in which side the green signal should be large during the traffic. The proposed work 
is to develop a system to recognize the vehicle number plate and retrieve the owner information from the database. It is based on inputting a vehicle number plate image to the system, which recognizes the characters of vehicle number plate. It matches the number plate with the respective data and then send the message to the owner of vehicle, whoever breaks the rule. The system we propose identify the density of traffic on individual lanes and thereby regulate the timing of the signals' timing and controls the crime.

\section{Literature Survey}

In the recent past, researchers have tested a wide array of technologies in an attempt to find improved methods of monitoring traffic conditions. A brief survey of technologies explored during the past decade and a half is given below to provide an understanding of the level of research interest in traffic surveillance technologies.

[1] R.B.Jayanthi Rajee, V. Devika, M. Abarna, A. Aswini Priya -"Traffic Management System using Image Processing and ARM Processor"- International Journal of Computer Systems (ISSN: 2394-1065), Volume 02- Issue 03, March, 2015.In this paper Image Processing technique used for traffic management system. Video sensors are used to capture the image sequences. Using Fuzzy constraint satisfaction methodology (FCM) the best time for traffic signal is calculated.This output is given to the ARM processor and the LED of the traffic signal is changed based on the ARM processor output.

[2] A Ranganath, T Sree Valli “ INTELLIGENT MANAGEMENT SYSTEM FOR DENSITY BASED CONTROL, STOLEN VEHICLE AND AUTO CLEARANCE "- International Journal of Advanced Technology in Engineering and Science, vol no.3Issue 08, August,2015. This paper proposed an advanced intelligent traffic control system. This system was implemented based on present criteria that tacking two condition in those one is heavy traffic control and another one is making a root for emergency vehicle like ambulance and VIP vehicle. A sensor network work is used to detect the traffic density, stolen vehicle and a RF based communication system for getting information from the emergency vehicle. To identify the stolen vehicle, RFID technology is used.

[3] Farheena Shaikh, Naisha Taban Khan, Saima Zareen Ansari "Controlling Traffic Light Signals to Implement Traffic Scheduling Using Density
Calculation" - International Journal on Recent and Innovation Trends in computing and communication, Volume: 3 Issue: 2 (ISSN: 2321-8169), September ,2014. The Traffic Management System using Density Calculation and Emergency Vehicle Alert, track traffic density at intersection using Road Side Unit (RSU) and Traffic Control Unit (TCU) will decide the timing of the traffic signal.

\section{Block diagram and components}

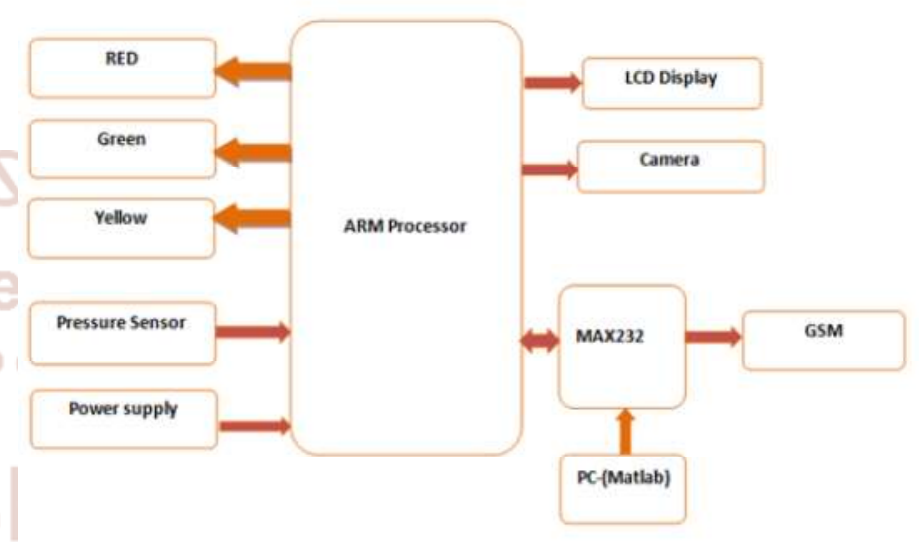

The ARM7TDMI-S is a general purpose 32-bit microprocessor, which offers high-performance and very low power consumption. This microcontroller used to make reliable in each component used in this project. When the signal gets RED then automatically the Piezo sensor should get activated, piezo sensor is connected with LPC 2138. The Timer is applied to signal for the proper orientation between each red and green signal. Signal 1,2,3,4 are interfaced with microcontroller, by using proper time the signals get activated one after another.

We have used Web Camera for this project. The use of camera is to capture the image of vehicle on pressure sense by the PIEZO sensor.Web camera is connected to Base station. The Resolution of this camera is MJPG_640X180. GSM is global system of mobile, by using GSM we can make communication between user and RTO office via wireless communication .

GSM is used for communication purpose. GSM interface with one base station to other base station. In this project we use GSM system for communication between RTO office to user mobile station. The basic step in the designing of any system is to design the power supply required for that system .In our project, we have designed 5V supply according to our need. 


\section{Prototype Implementation:}

\section{A. Algorithm:}

Step1: Start the program.

Step2: Read the input background image of the empty road.

Step3: Read the new image with vehicles on road.

Step4: Convert the images to grayscale format using double precision.

Step5: Find the width and height of the image.

Step6: Set threshold value $=$

Step7: Find the difference between frames based on the threshold.

Step8: If frame diff $>$ than 44 , then assign that image to a variable else " 0 " if no difference is found.

Step9: Increase the contrast of the output image using imadjust()

Step10:Find a greythreshold value using graythresh() command.

Step11: Add Gaussian noise to the output difference.

Step12: Apply Weiner filter to filter the blobs

Step13: Convert to binary image

Step14: Fill holes to the blobs

Step15: Open all blobs having area greater than 5000

Step16: Count the number of cars using bwconncomp or bwlabel

Step17: Display the output image

Step18: Stop the program

\section{B. Implementation:}

The system is designed to develop a vehicle density based traffic management system. The camera captures the image of traffic condition at certain interval of time. Decision is taken by the traffic light engine depending on the density of vehicles present. The image captured in the traffic signal is processed and converted into grayscale image then its threshold is calculated based on which the contour has been drawn in order to calculate the number of vehicles present in the image. After calculating the number of vehicles we will came to know in which side the density is high. The time period of green light and red light is assigned on the basis of the density of the traffic present at that time.

Automatic Number Plate Recognition (ANPR) technique offers the ability to find a person who breaks the Traffic rules and give notice via mobile phone. This process is done via pressure sensor which is activated when red signal is $\mathrm{ON}$, whenever any vehicle crosses Zebra crossing line, sensor gives signal to digital web camera. Camera can be used to identify vehicles by reading number plates and use the information that is stored at the RTO server.Then, the message is sent to the respective owner of the vehicle using GSM technique.

\section{Result 1:}

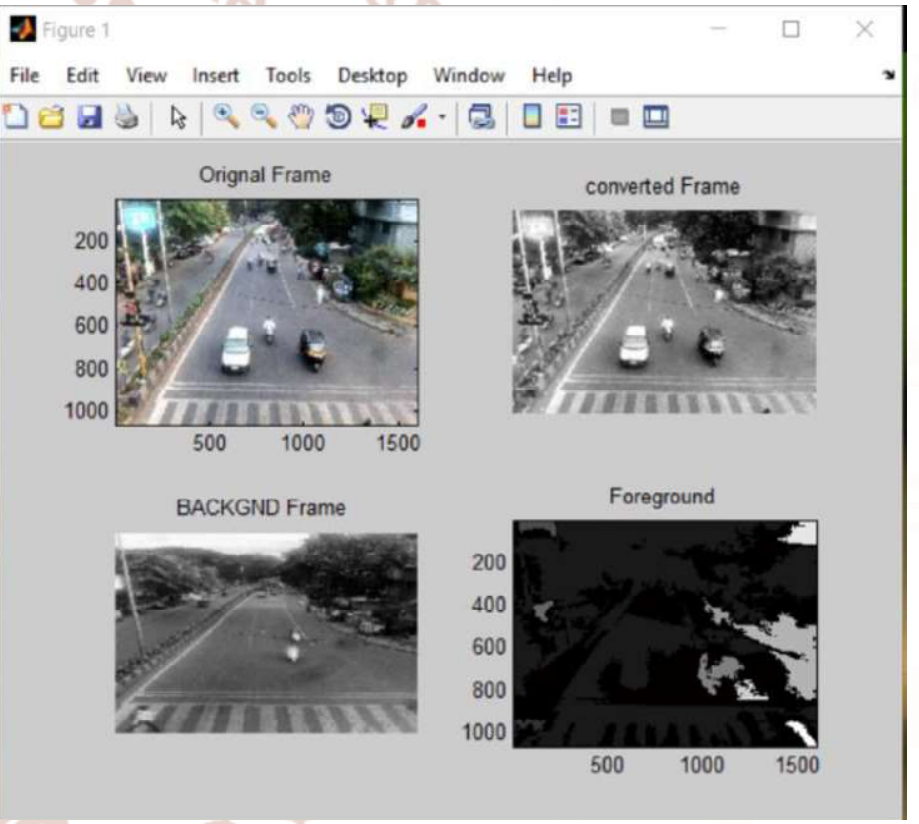

Command Window

(i) New to MATLAB? Watch this Video see Examples, or read Getting Started.

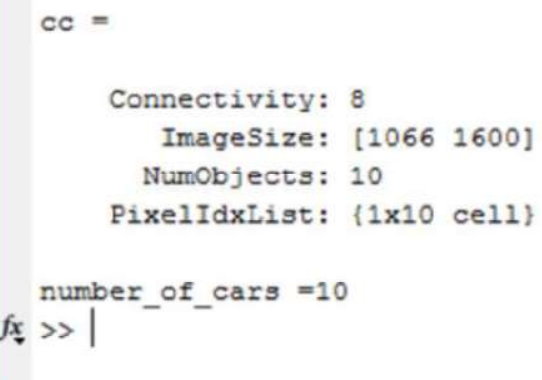


D. Result 2:
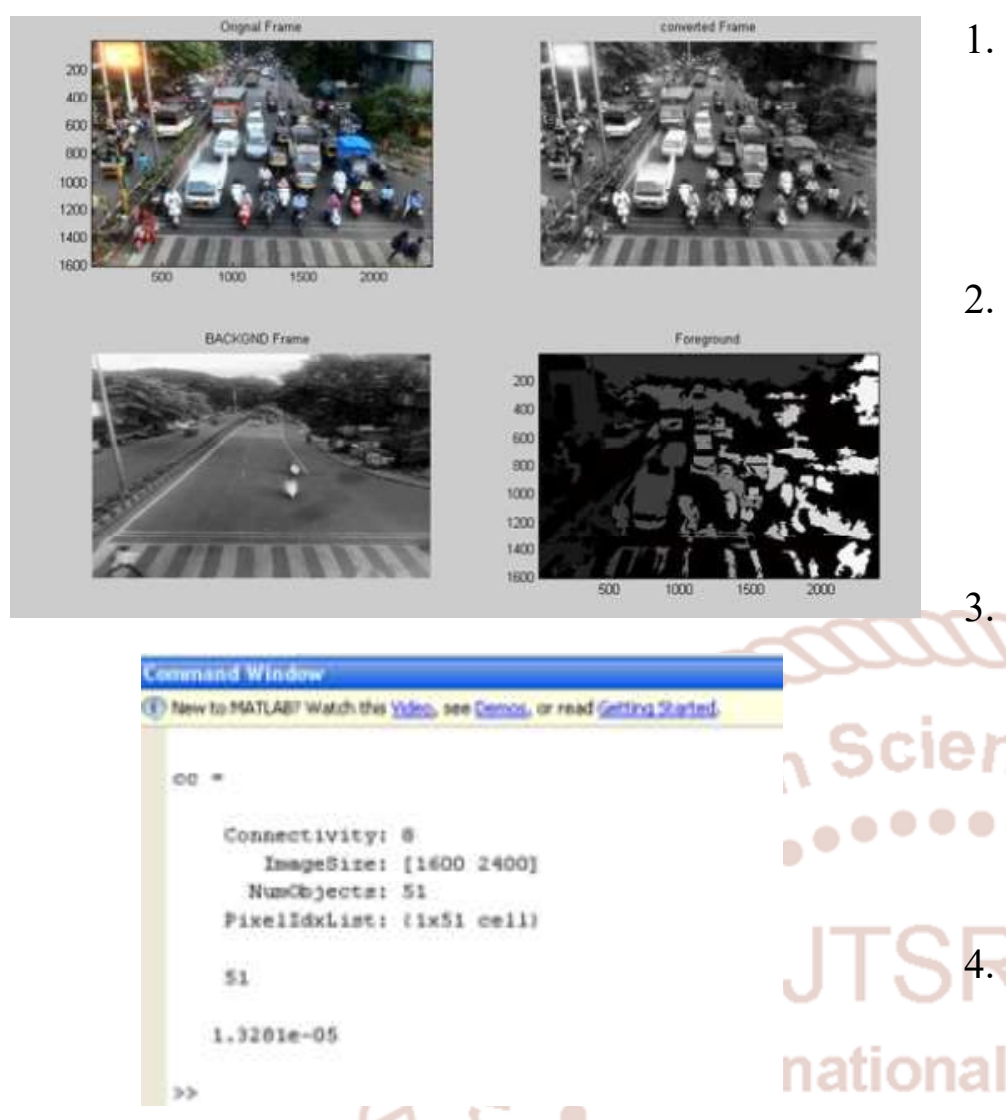

\section{Conclusion:}

To reduce the congestion and crime an advanced system is designed here in this project. In the earlier system, switching of signal at the particular instant of time. But in our system switching time depend upon the vehicle density of traffic. The ANPR technique used to identify vehicles by only their number plates on Top. ANPR technology tends to be region-specific, owing to plate variation from place to place. However, as we have developed, the systems have become much more accurate and reliable. Using this technique RTO police can fine the person who disobey the traffic rule i.e. signal break. We believe that this may bring a revolutionary change in traffic management system on its application in its actual field environment.

\section{REFERENCES:}

1. R.B.Jayanthi Rajee, V. Devika, M. Abarna, A. Aswini Priya -"Traffic Management System using Image Processing and ARM Processor"International Journal of Computer Systems (ISSN: 2394-1065), Volume 02- Issue 03, March, 2015.

2. A Ranganath, T Sree Valli “ INTELLIGENT MANAGEMENT SYSTEM FOR DENSITY BASED CONTROL, STOLEN VEHICLE AND AUTO CLEARANCE "- International Journal of Advanced Technology in Engineering and Science,vol no.3-Issue 08, August,2015.

3. Farheena Shaikh, Naisha Taban Khan, Saima Zareen Ansari "Controlling Traffic Light Signals to Implement Traffic Scheduling Using Density Calculation" - International Journal on Recent and Innovation Trends in computing and communication, Volume: 3 Issue: 2 (ISSN: 2321 8169), September ,2014.

4. Mahesh Satpute, Nikhil Nanhe, Ashish Nanda, Amit Patil and Pradnyashil Kelkar- "Survey on Intelligent Traffic Signal Management System" International Journal For Research in Emerging Science and Technology, Special-Issue-1-Jan2017.

5. Vismay Pandit, Jinesh Doshi, Dhruv Mehta, Ashay Mhatre and Abhilash Janardhan "Smart traffic control system using Image Processing " International Journal of Emerging Trends and Technology in Computer Science, vol no.3, Issue1, January and February 2014. 